\title{
Insight on the Pro-oxidant Capability of Amphotericin B in Lipid Media: A Theoretical Study
}

\author{
Manuel E. Medina ${ }^{1}$, Thuluz Meza-Menchaca ${ }^{1}$, and Angel Trigos ${ }^{1}$ \\ ${ }^{1}$ Universidad Veracruzana
}

July 23, 2020

\begin{abstract}
A study of the pro-oxidant capacity of amphotericin B was carried out by analyzing the photosensitizing ability of an amphotericin B model. Although amphotericin B is a well-known broad-spectrum antibiotic, little is known about the mechanism of its pro-oxidant capability. This study employed density functional theory to assess the photosensitizing capacity of AMP. Furthermore, electron transfer reactions were modeled according to the Marcus theory, considering that lipid media can mimic the environment of the cell membrane. The results show that AMP is a pro-oxidant molecule mainly through the type II mechanism, with the corresponding production of 1O2. In this way, AMP could induce oxidative stress because it is capable of generating reactive oxygen species. In this case, AMP showed a reaction rate constants of $8.94 \times 109$ and $1.89 \times 1010$ M-1 s-1 for the type I and II mechanisms, respectively, while the overall reaction rate constant was $2.79 \times 1010$ M- 1 s- 1 . Therefore, the results of this study support the pro-oxidant capacity of amphotericin B in lipid media, and considering that ergosterol is more susceptible to undergoing oxidative damage by reactive oxygen species than cholesterol, this mechanism could contribute to the antifungal activity of amphotericin B.
\end{abstract}

\section{Introduction}

Reactive oxygen species (ROS) are a group of fundamental molecules in organisms. They can be neutral as ${ }^{1} \mathrm{O}_{2}$, ionic as $\mathrm{O}_{2}{ }^{*}{ }^{-}$or radicals as ${ }^{*} \mathrm{OOH}$. However, under oxidative stress, these molecules are capable of causing damage to an organism through oxidation reactions in biological molecules of physiological importance, for example, proteins ${ }^{1}$ lipids $^{2}$, and DNA. ${ }^{3}$ Oxidative stress is the imbalance between the generation and consumption of ROS and is associated with widely distributed diseases, especially cancer, ${ }^{4}$ lung disorders, ${ }^{5}$ cardiovascular disorders, and atherosclerosis. ${ }^{6}$

Pro-oxidant molecules are any endobiotic or xenobiotic that produces oxidative stress, either by providing oxidative species such as ROS or by inhibiting the physiological mechanisms responsible for reducing the ROS concentration in the organism. ${ }^{7}$ Among pro-oxidant are found photosensitizers, which are molecules that can produce and increase the level of ROS and generate oxidative stress through two main mechanisms. These molecules can produce ROS through the type I mechanism, where an $\mathrm{O}_{2}{ }^{*}$ molecule is provided through the electron transfer mechanism from the photosensitizer to the oxygen molecule or through the type II mechanism, where ${ }^{1} \mathrm{O}_{2}$ is obtained by transferring the excitation energy from the photosensitizer to oxygen molecule. ${ }^{8,9}$

Amphotericin B is a heptaene macrolide drug that is used as a broad-spectrum antibiotic. It has been considered for several decades as the standard for the treatment of systemic fungal and yeast infections. Several mechanisms have been proposed to explain the antifungal activity of amphotericin B. The most studied mechanism regarding antifungal activity is when amphotericin B interacts with ergosterol in the fungal cell membrane; in this way, a channel that connects the intracellular and extracellular medium through the cell membrane is formed and thereby promoting ions exchange that causes disruption of cellular homeostasis 
and consequently leads to cell death. ${ }^{10,11,12}$ The selectivity observed between the cell membranes of animals and fungi is based on higher intermolecular interactions between amphotericin B and ergosterol (in fungi) than the former with cholesterol (in animals). ${ }^{13,14}$ However, a second mechanism has been proposed that enables us to understand the antibiotic property of amphotericin B. In this mechanism, it was suggested that this molecule can act as a pro-oxidant molecule and produce oxidative stress through the generation of ROS. ${ }^{15}$ As observed in a study in which Aspergillus terreus was used (as it is resistant to amphotericin B), it was reported that the level of catalase production in $A$. terreus was significantly higher than in $A$. fumigatus (non-resistant to amphotericin B); this higher production level may contribute to amphotericin $\mathrm{B}$ resistance in A. terreus, since oxidative damage has been implicated in the action of amphotericin $\mathrm{B} ;^{16}$ in addition, singlet oxygen $\left({ }^{1} \mathrm{O}_{2}\right)$ generation of amphotericin $\mathrm{B}$ under UVA was only below to nalidixic acid in a study on the production of singlet oxygen in a group of antiobiotics; ${ }^{17}$ in the same sense, the intracellular induction of ROS was determined in different pathogenic yeast species and it was found that amphotericin B induces the formation of ROS in all the species tested; hence, the data demonstrate that the production of ROS by amphotericin B is an essential mechanism of action that correlates with the fungicidal effect. ${ }^{18}$ The observed selectivity between fungi and animals in this second mechanism is based on a higher susceptibility to oxidative damage by ROS of ergosterol, compared to cholesterol in the cell membrane. ${ }^{19-21}$<smiles>CC(O)C(C)/C=C/C=C/C=C/C=C/C=C/C=C/C=C/[C@@H](CC1O[C@](O)(CC(O)CC(O)C(O)CCC(O)CC(O)CC(=O)OC(C)C(C)C(O)C(C)O)CC(O)[C@H]1C(=O)O)OC1OC(C)C(O)C(N)C1O</smiles>

Amphotericin B

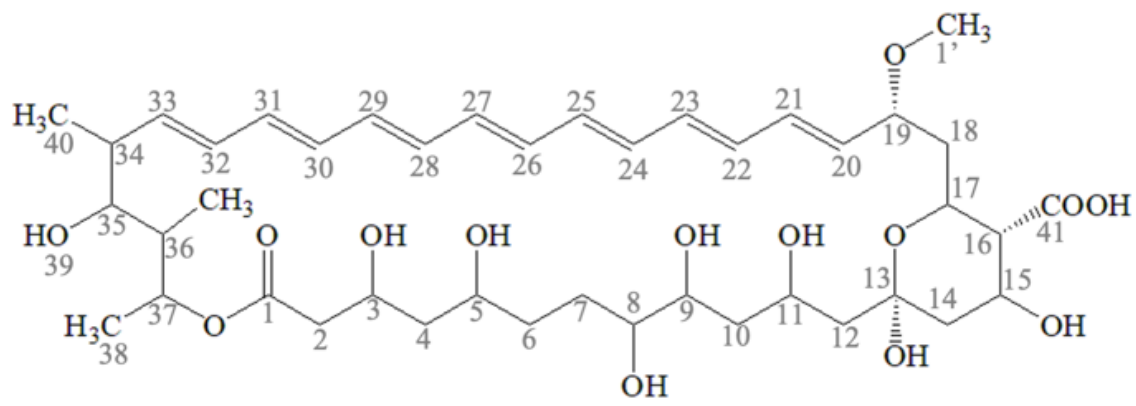

AMP

According to the above, in this work, the study of the photosensitizing capacity of amphotericin B was performed using a molecular model of amphotericin B; this is shown in Figure 1. The single-electron transfer mechanism was considered in the reaction between AMP, its oxidized, and reduced forms with oxygen and superoxide molecules. Finally, thermodynamic and kinetic studies in lipid media on amphotericin B were reported for the first time.

Figure 1. Amphotericin B and the model (AMP) employed in this study. 


\section{Computational methods}

All the calculations were carried out with the Gaussian 16 program package, ${ }^{22}$ employing the M06-2X and the $6-31 \mathrm{G}(\mathrm{d}, \mathrm{p})$ basis set, all the geometries were fully optimized, and their frequency calculations were carried out. ${ }^{23}$ Pentylethanoate was considered as the lipid medium to mimic the membrane cell environment, using (SMD) the continuum solvation model density. ${ }^{24}$ The M06-2X functional has been recommended by its developers to perform kinetic calculations and is reported to be one of the best performing functionals for kinetic calculations in solutions. ${ }^{25,26}$ SMD is a solvation model that could be employed to any charged or uncharged solute in a liquid medium. ${ }^{24}$ The geometry optimization and frequency calculations, including the solvent effect by the first excited-state singlet were performed employing the TD-DFT calculations at the TD-M06-2X/6-31G(d,p) level. The energy values were improved by a single point calculation at the M06-2X/6-311++G(d,p) level.

The conventional transition state theory (TST) was used to estimate the reaction rate constant, according to the following: ${ }^{27,28}$

$k=\frac{k_{B} T}{h} e^{-G^{\neq} / R T}(1)$

where $k_{B}, h$, and $R$ are the Boltzmann, Planck, and gas constants, respectively; additionally, $T$ and $\Delta G$ [?] are the temperature and the Gibbs free energy of activation, respectively.

The single electron transfer mechanism (SET) was considered in all the reactions, and the Gibbs free energy of reaction $([?] \mathrm{G})$ was calculated from separate reactants and products of each SET reaction; as well as, the Gibbs free energy of activation ([?]GG ${ }^{[?]}$ ) was obtained employing Marcus theory. ${ }^{29-31}$ The Gibbs free energy of activation was described based on the Gibbs free energy of reaction $([?] \mathrm{G})$ and the nuclear reorganization energy $(\lambda)$.

$G^{\neq}=\frac{\lambda}{4}\left(1+\frac{\Delta G}{\lambda}\right)^{2}(2)$

The nuclear reorganization energy $(\lambda)$ was obtained according to the following:

$\lambda=\Delta E-\Delta G(3)$

thus, [?] $E$ was the difference of no adiabatic energies between reactants and vertical products. This estimate is comparable to that suggested for intramolecular electron exchange reactions. ${ }^{32}$

The apparent rate constant cannot be directly obtained from TST calculations when calculated reaction rate constants values are closer to, or within the diffusion-limit regime; hence, Collins-Kimbal theory was used: ${ }^{33}$

$k_{\text {app }}=\frac{k_{D} k}{k_{D}+k}$

where $k$ is the thermal reaction rate constant and was obtained from transition state theory calculations and $k_{D}$ is the steady-state Smoluchowski rate constant in an irreversible bimolecular diffusion-controlled reaction, ${ }^{34}$ and was calculated according to the following equation:

$k_{D}=4 \pi R D_{\mathrm{AB}} N_{A}(4)$

where $R$ is the reaction distance, $D_{A B}$ is the mutual diffusion coefficient of the reactants $\mathrm{A}$ and $\mathrm{B}$, and $N_{A}$ is the Avogadro number. $D_{A B}$ has been calculated from $D_{A}$ and $D_{B} \cdot{ }^{35}$ The Stoke-Einstein approach was employed to estimate $D_{A}$ and $D_{B},{ }^{36,37}$ according to the following equation:

$D=\frac{k_{B} T}{6 \pi \eta-1}(5)$

where $k_{\mathrm{B}}$ and $T$ are the Boltzmann constant and temperature, respectively, while the $\eta$ is the viscosity of the solvent, in this case, pentylethanoate $\left(\eta=8.62 \times 10^{-4} \mathrm{~Pa} \mathrm{~s}\right)$, and the radius of the solute - .

In the kinetic study, the endergonic reaction paths were not included because such reactions would be reversible; as a consequence, the corresponding products would not be observed. The methodology employed 
in this study is in line with the quantum mechanics-based test for overall free radical scavenging activity (QM-ORSA); besides, this methodology has been verified by contrast with experimental results. ${ }^{38}$

\section{Results and discussion}

The study on the pro-oxidant capability of AMP was carried out through the calculation of excitation energies. The results calculated show that AMP has a maximum wavelength of $399.32 \mathrm{~nm}$ in pentylethanoate and this result was corroborated with the experimental results, where the maximum wavelength for amphotericin $\mathrm{B}$ was $408 \mathrm{~nm}$ in a non-polar media. ${ }^{39}$

Additionally, the main characteristic bond distances of AMP and its reaction intermediates such as ${ }^{1} \mathrm{AMP},{ }^{1} \mathrm{AMP}^{*},{ }^{3} \mathrm{AMP}, \mathrm{AMP}^{*}$-and $\mathrm{AMP}^{*+}$, are shown in Table 1 , as well as the numbering of atoms are shown in Figure 1. In these were observed that C20-C21, C22-C23, C24-C25, C26-C27, C28-C29, C30- C31, and $\mathrm{C} 32-\mathrm{C} 33$ bond distances are larger in the ${ }^{1} \mathrm{AMP}^{*},{ }^{3} \mathrm{AMP}, \mathrm{AMP}^{*}$ - and $\mathrm{AMP}^{*+}$ species than ${ }^{1} \mathrm{AMP}$. It was also observed that the largest bond corresponds to the ${ }^{3} \mathrm{AMP}$ molecule. A decrease in bond distances C21-C22, C23-C24, C25-C26, C27-C28, C29-C30, and C31-C32 were seen in ${ }^{1} \mathrm{AMP}^{*},{ }^{3} \mathrm{AMP}, \mathrm{AMP}^{*}$ - and $\mathrm{AMP}^{*+}$ when compared with the ${ }^{1} \mathrm{AMP}$. The species that showed the shortest bond distance is ${ }^{3} \mathrm{AMP}$.

Table 1. Bond distances in angstroms from AMP and their reaction intermediates in lipid media, at 298 K.

\begin{tabular}{llllll}
\hline Distance & ${ }^{1}$ AMP & ${ }^{1}$ AMP $^{*}$ & ${ }^{3}$ AMP & AMP $^{*}$ & AMP $^{*+}$ \\
\hline C20-C21 & 1.340 & 1.353 & 1.355 & 1.348 & 1.351 \\
C21-C22 & 1.453 & 1.429 & 1.423 & 1.440 & 1.429 \\
C22-C23 & 1.350 & 1.381 & 1.397 & 1.371 & 1.375 \\
C23-C24 & 1.446 & 1.402 & 1.382 & 1.417 & 1.407 \\
C24-C25 & 1.352 & 1.403 & 1.432 & 1.389 & 1.389 \\
C25-C26 & 1.445 & 1.387 & 1.359 & 1.402 & 1.397 \\
C26-C27 & 1.353 & 1.412 & 1.446 & 1.397 & 1.393 \\
C27-C28 & 1.444 & 1.387 & 1.358 & 1.400 & 1.399 \\
C28-C29 & 1.352 & 1.404 & 1.433 & 1.393 & 1.385 \\
C29-C30 & 1.446 & 1.400 & 1.380 & 1.411 & 1.412 \\
C30-C31 & 1.350 & 1.383 & 1.399 & 1.376 & 1.370 \\
C31-C32 & 1.452 & 1.427 & 1.421 & 1.435 & 1.434 \\
C32-C33 & 1.342 & 1.356 & 1.358 & 1.352 & 1.350 \\
\hline
\end{tabular}

The pro-oxidant ability study of AMP was carried out through the analysis of its photosensitive capacity employing the electron transfer reactions. The reaction modeled involving AMP, its derivatives and the ${ }^{3} \mathrm{O}_{2}$ and $\mathrm{O}_{2}{ }^{*}$ - were also considered, as molecules that can be found in high concentration in the organism, according to the following reactions:

\begin{tabular}{|c|c|}
\hline${ }^{1} \mathrm{AMP}+{ }^{3} \mathrm{O}_{2}-\mathrm{AMP}^{*+}+\mathrm{O}_{2}{ }^{*-}$ & $(\mathrm{Rxn} 1)$ \\
\hline${ }^{1} \mathrm{AMP}^{*}+{ }^{3} \mathrm{O}_{2}-\mathrm{AMP}^{*+}+\mathrm{O}_{2}{ }^{*}-$ & $(\operatorname{Rxn} 2)$ \\
\hline${ }^{3} \mathrm{AMP}+{ }^{3} \mathrm{O}_{2}-\mathrm{AMP}^{*+}+\mathrm{O}_{2}{ }^{*}-$ & (Rxn3) \\
\hline${ }^{1} \mathrm{AMP}+\mathrm{O}_{2}{ }^{{ }^{*}}-\mathrm{AMP}^{*_{-}}+{ }^{1} \mathrm{O}_{2}$ & $(\mathrm{Rxn} 4)$ \\
\hline${ }^{1} \mathrm{AMP}+\mathrm{O}_{2}{ }^{{ }^{*}-}-\mathrm{AMP}^{*-}+{ }^{3} \mathrm{O}_{2}$ & $(\mathrm{Rxn} 5)$ \\
\hline${ }^{1} \mathrm{AMP}^{*}+\mathrm{O}_{2}{ }^{*-}-\mathrm{AMP}^{*-}+{ }^{1} \mathrm{O}_{2}$ & (Rxn6) \\
\hline${ }^{1} \mathrm{AMP}^{*}+\mathrm{O}_{2}{ }^{*_{-}}-\mathrm{AMP}^{*_{-}}+{ }^{3} \mathrm{O}_{2}$ & $(\mathrm{Rxn} 7)$ \\
\hline${ }^{3} \mathrm{AMP}+\mathrm{O}_{2}{ }^{{ }^{*}-}-\mathrm{AMP}^{*}{ }_{-}+{ }^{1} \mathrm{O}^{2}$ & $(\mathrm{Rxn} 8)$ \\
\hline${ }^{3} \mathrm{AMP}+\mathrm{O}_{2}{ }^{{ }^{-}}-\mathrm{AMP}^{*-}+{ }^{3} \mathrm{O}_{2}$ & (Rxn9) \\
\hline${ }^{1} \mathrm{AMP}+{ }^{1} \mathrm{AMP}-\mathrm{AMP}^{*+}+\mathrm{AMP}$ & $(\mathrm{Rxn} 10$ \\
\hline
\end{tabular}




\begin{tabular}{|c|c|}
\hline${ }^{1} \mathrm{AMP}+{ }^{3} \mathrm{O}_{2}-\mathrm{AMP}^{*+}+\mathrm{O}_{2}{ }^{*-}$ & $(\mathrm{Rxn} 1)$ \\
\hline${ }^{1} \mathrm{AMP}+{ }^{1} \mathrm{AMP}^{*}-\mathrm{AMP}^{*+}+\mathrm{AMP}^{*-}$ & (Rxn11) \\
\hline${ }^{1} \mathrm{AMP}+{ }^{3} \mathrm{AMP}-\mathrm{AMP}^{*+}+\mathrm{AMP}^{*-}$ & $(\mathrm{Rxn} 12)$ \\
\hline${ }^{1} \mathrm{AMP}^{*}+{ }^{1} \mathrm{AMP}^{*}-\mathrm{AMP}^{*+}+\mathrm{AMP}^{*}$ & (Rxn13) \\
\hline${ }^{1} \mathrm{AMP}^{*}+{ }^{3} \mathrm{AMP}-\mathrm{AMP}^{*+}+\mathrm{AMP}^{*}$ & (Rxn14) \\
\hline${ }^{3} \mathrm{AMP}+{ }^{3} \mathrm{AMP}-\mathrm{AMP}^{*+}+\mathrm{AMP}^{*-}$ & $(\mathrm{Rxn} 15)$ \\
\hline $\mathrm{AMP}^{*+}+\mathrm{O}_{2}{ }^{*_{-}}-{ }^{1} \mathrm{AMP}+{ }^{1} \mathrm{O}_{2}$ & $(\mathrm{Rxn} 16)$ \\
\hline $\mathrm{AMP}^{*+}+\mathrm{O}_{2}{ }^{{ }_{-}}-{ }^{1} \mathrm{AMP}+{ }^{3} \mathrm{O}_{2}$ & $(\mathrm{Rxn} 17)$ \\
\hline $\mathrm{AMP}^{*+}+\mathrm{O}_{2}{ }^{{ }_{-}-}{ }^{3} \mathrm{AMP}+{ }^{1} \mathrm{O}_{2}$ & (Rxn18) \\
\hline $\mathrm{AMP}^{*+}+\mathrm{O}_{2}{ }^{{ }_{-}-}-{ }^{3} \mathrm{AMP}+{ }^{3} \mathrm{O}_{2}$ & (Rxn19) \\
\hline $\mathrm{AMP}^{*-}+{ }^{3} \mathrm{O}_{2}-{ }^{1} \mathrm{AMP}+\mathrm{O}_{2}{ }^{*-}$ & $(\mathrm{Rxn} 20)$ \\
\hline $\mathrm{AMP}^{*_{-}}+{ }^{3} \mathrm{O}_{2}-{ }^{3} \mathrm{AMP}+\mathrm{O}_{2}{ }^{{ }_{-}}$ & $(\mathrm{Rxn} 21)$ \\
\hline
\end{tabular}

In Table 2 are shown the thermodynamic results of the photosensitizing ability of AMP in lipid media. All reactions considered in the electronic transfer involved in the photosensitizing ability of AMP in the presence of oxygen molecules were modeled.

The results showed that Rxn6, Rxn7, Rxn9, Rxn13, Rxn14, Rxn16, Rxn17, Rxn19 and Rxn20 reactions were exergonic with the following Gibbs free energies -20.03, -57.94, -22.30, -57.64, -22.00, -22.57, -60.48, -35.94 and $-2.24 \mathrm{kcal} / \mathrm{mol}$, respectively. The results obtained showed that both reaction mechanisms are present in the reaction routes considered. It was observed that reaction Rxn20 yields the ${ }^{1} \mathrm{AMP}$ and $\mathrm{O}_{2}{ }^{{ }^{-}}$molecules, following $\mathrm{AMP}^{*-}+{ }^{3} \mathrm{O}_{2}$ reagents, and this reaction mechanism supports mechanism type I, while reactions Rxn6 and Rxn16 where ${ }^{1} \mathrm{O}_{2}$ occurred, following reagents ${ }^{1} \mathrm{AMP}^{*}+\mathrm{O}_{2}{ }^{{ }_{-}}$and $\mathrm{AMP}^{*+}+\mathrm{O}_{2}{ }^{{ }^{*}}$, respectively are involved in the type II mechanism.

According to thermodynamic results, AMP can produce ROS by both mechanisms types I and II, in lipid media. Therefore, in the kinetic study, the exergonic routes of the reaction were analyzed. For this reason, the kinetic study involved Rxn6, Rxn7, Rxn9, Rxn13, Rxn14, Rxn16, Rxn17, Rxn19, and Rxn20 reactions.

The study of the pro-oxidant capability of AMP was carried out through kinetic analysis. These were carried out in the exergonic reactions obtained in the thermodynamic results.

Table 2 . Gibbs free energy of reaction $(\Delta \mathrm{G}, \mathrm{kcal} / \mathrm{mol})$ in the reactions considering the photosensitizing activity of AMP in lipid media, at $298 \mathrm{~K}$.

\begin{tabular}{llll}
\hline Reaction & $\Delta \mathrm{G}$ & Reaction & $\Delta \mathrm{G}$ \\
\hline Rxn1 & 60.48 & Rxn12 & 38.18 \\
Rxn2 & 0.30 & Rxn13 & -57.64 \\
Rxn3 & 35.94 & Rxn14 & -22.00 \\
Rxn4 & 40.15 & Rxn15 & 13.64 \\
Rxn5 & 2.24 & Rxn16 & -22.57 \\
Rxn6 & -20.03 & Rxn17 & -60.48 \\
Rxn7 & -57.94 & Rxn18 & 1.97 \\
Rxn8 & 15.61 & Rxn19 & -35.94 \\
Rxn9 & -22.30 & Rxn20 & -2.24 \\
Rxn10 & 62.72 & Rxn21 & 22.30 \\
Rxn11 & 2.54 & & \\
\hline
\end{tabular}

Accordingly, the results of the kinetics study are shown in Table 3. These results indicate that reactions Rxn6, Rxn9, Rxn16, Rxn19, and Rxn20 have the smallest Gibbs free energy of activation. Consequently, 
they show values of reaction rate constants limited by the diffusion rate; all these reactions show a $k$ app to the order of $10^{9} \mathrm{M}^{-1} \mathrm{~s}^{-1}$. The reaction route that supports the type I mechanism was Rxn20, shown a reaction rate constant of $8.94 \times 10^{9} \mathrm{M}^{-1} \mathrm{~s}^{-1}$, while the Rxn6 and Rxn16 reactions support the type II mechanism, which has reaction rate constants of $9.40 \times 10^{9}$ and $9.53 \times 10^{9} \mathrm{M}^{-1} \mathrm{~s}^{-1}$, respectively.

Table 3 . Gibbs free energy of activation ([?] $\left.G^{[?]}, \mathrm{kcal} / \mathrm{mol}\right), \lambda(\mathrm{kcal} / \mathrm{mol})$, diffusion rate constant $\left(k_{a p p}\right.$, $\left.\mathrm{M}^{-1} \mathrm{~S}^{-1}\right)$, and apparent rate constant $\left(k_{a p p}, \mathrm{M}^{-1} \mathrm{~s}^{-1}\right)$ of the photosensitizer capacity of AMP in lipid media, at $298 \mathrm{~K}$.

\begin{tabular}{lllll}
\hline Reacción & {$[?] G^{[?]}$} & $\lambda$ & $k_{\mathrm{D}}$ & $k_{\text {app }}$ \\
\hline Rxn6 & 0.11 & 17.29 & $9.41 \times 10^{9}$ & $9.40 \times 10^{9}$ \\
Rxn7 & 22.87 & 17.70 & $9.41 \times 10^{9}$ & $1.06 \times 10^{-4}$ \\
Rxn9 & 0.06 & 20.11 & $9.60 \times 10^{9}$ & $9.58 \times 10^{9}$ \\
Rxn13 & 259.03 & 2.89 & $7.67 \times 10^{9}$ & $8.33 \times 10^{-178}$ \\
Rxn14 & 13.14 & 5.31 & $7.67 \times 10^{9}$ & $1.46 \times 10^{3}$ \\
Rxn16 & 0.03 & 20.98 & $9.55 \times 10^{9}$ & $9.53 \times 10^{9}$ \\
Rxn17 & 17.85 & 21.40 & $9.55 \times 10^{9}$ & $5.15 \times 10^{-1}$ \\
Rxn19 & 1.22 & 24.93 & $9.55 \times 10^{9}$ & $9.43 \times 10^{9}$ \\
Rxn20 & 2.57 & 14.41 & $1.00 \times 10^{10}$ & $8.94 \times 10^{9}$ \\
\hline
\end{tabular}

The other reactions considered in the kinetic study were slow, and as a consequence, these reactions do not contribute to the photosensitizing capability of AMP.

Additionally, the calculations were performed to obtain the total rate constant for each type I and II mechanisms $\left(k_{\text {typeI }}\right.$ and $\left.k_{\text {typeII }}\right)$ in the photosensitizing activity of AMP in lipid media, according to the next equations:

$k_{\text {typeI }}=k_{R x n 20}(7)$
$k_{\text {typeII }}=k_{R x n 6}+k_{R x n 16}(8)$
$k_{\text {overall }}=k_{\text {typeI }}+k_{\text {typeII }}(9)$

The overall reaction rate constants calculated from the equations above are shown in Table 4 . It was observed that the type II mechanism was the fastest, and in the photosensitive capacity of AMP, this mechanism was preferred by $67.9 \%$. In contrast, the type I mechanism is carried out in $32.1 \%$. AMP has a photosensitive capacity through both mechanisms; however, the primary mechanism on the photosensitizing ability of AMP in lipid media was type II. Finally, the overall reaction rate constant calculated on the photosensitizing capacity of AMP in lipid media was $2.79 \times 10^{10} \mathrm{M}^{-1} \mathrm{~s}^{-1}$.

Table 4 : Reaction rate constants and branching ratios $(\Gamma, \%)$ of the photosensitizing capacity of $A M P$ in lipid media, at $298 \mathrm{~K}$.

\begin{tabular}{lll}
\hline Mechanism & $k\left(\mathrm{M}^{-1}, \mathrm{~s}^{-1}\right)$ & $\Gamma$ \\
\hline Type I & $8.94 \times 10^{9}$ & 32.1 \\
Type II & $1.89 \times 10^{10}$ & 67.9 \\
Overall & $2.79 \times 10^{10}$ & \\
\hline
\end{tabular}




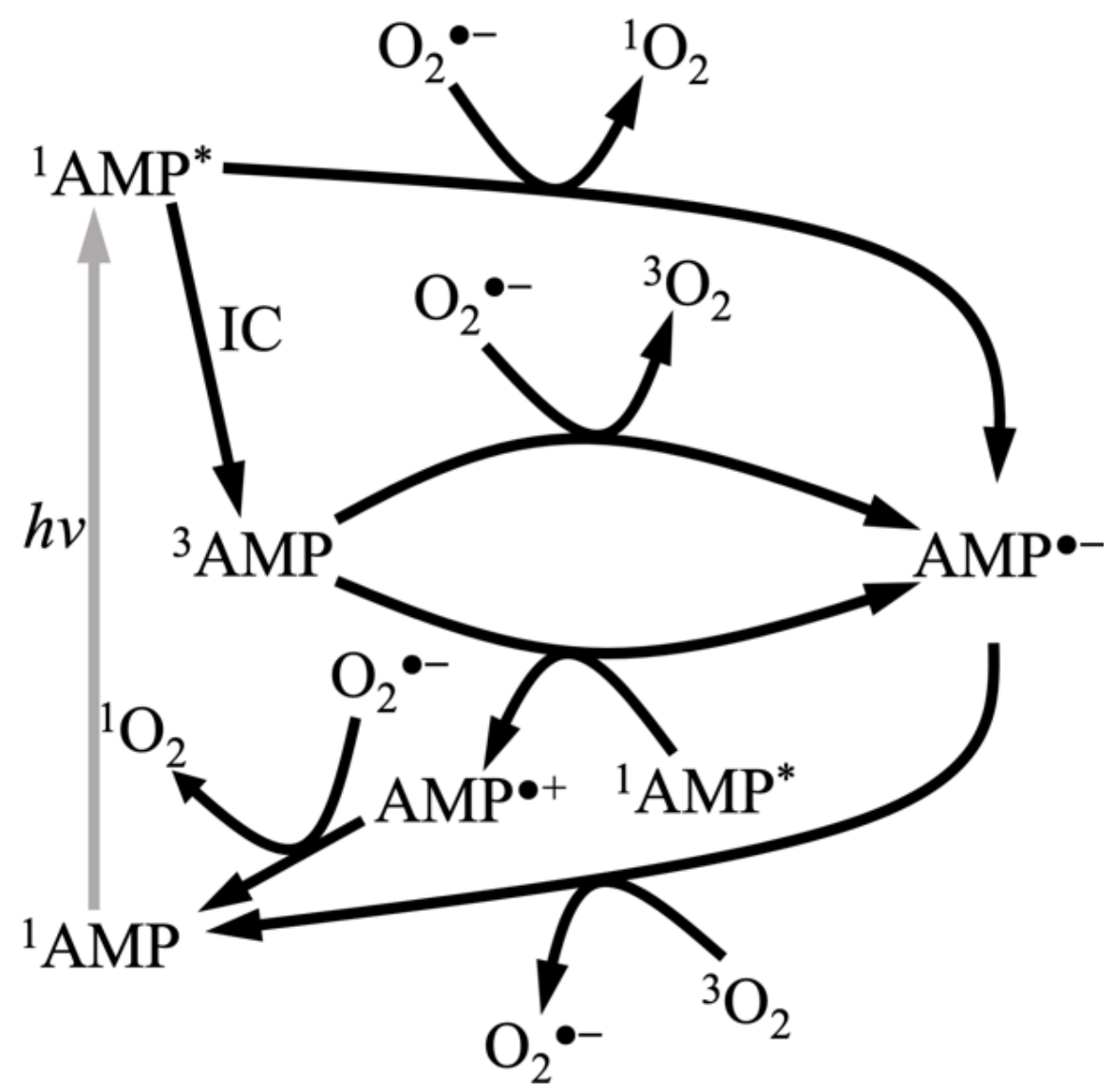

Moreover, Figure 2 shows the main reactions in the photosensitizing capacity of AMP in lipid media. In this, two cyclic routes could be observed. In the first ${ }^{1}$ AMP-AMP ${ }^{*}-\mathrm{AMP}^{*}-{ }^{1} \mathrm{AMP}$ and in the second ${ }^{1} \mathrm{AMP}-$ ${ }^{1} \mathrm{AMP}^{*}{ }^{3} \mathrm{AMP}-\mathrm{AMP}^{*+}{ }_{-}{ }^{1} \mathrm{AMP}$ found, both reaction routes carried out the regeneration of AMP in lipid media. According to the above, AMP can yield ROS by a cyclic path of reactions in lipid media and produce oxidative stress.

Figure 2. Reactions involved in the photosensitizing capability of AMP in lipid media.

In summary, the results concerning the photosensitizing capability of AMP showed that the main differences between AMP and their derivatives are in the electronic conjugation of their double bonds; hence, the photosensitizing property of AMP could be attributed to the presence of electronic conjugation of their double bonds. Consequently, we might propose that amphotericin B can possess the pro-oxidant capability in lipid media, through the photosensitizing property. In the same way, amphotericin B can produce ROS by a cyclic reaction that could regenerate amphotericin B. Therefore, this study supports the pro-oxidant capability of amphotericin B in lipid media and contributes to knowledge about the primary mechanism involved in its antibiotic property.

\section{Conclusion}

The study of the pro-oxidant capability of amphotericin B was carried out through the use of an amphotericin B model AMP. The AMP photosensitizer study was performed employing the density functional theory. In this study, the electron transfer mechanism was based on the Marcus theory, which considers the lipid medium to mimic the environment of the cell membrane. Thermodynamic results show that AMP can be a pro-oxidant molecule through types I and II mechanisms. The kinetic results indicate that AMP is a pro-oxidant molecule mainly through the type II mechanism. The AMP showed a reaction rate constant 
for the types I and II mechanisms of $8.94 \times 10^{9}$ and $1.89 \times 10^{10} \mathrm{M}^{-1} \mathrm{~s}^{-1}$, respectively, while the overall reaction rate constant was $2.79 \times 10^{10} \mathrm{M}^{-1} \mathrm{~s}^{-1}$. Therefore, the results of this study support the pro-oxidant capability of amphotericin B in lipid media and considering that ergosterol is more susceptible to undergoing oxidative damage by ROS than cholesterol, this mechanism could contribute toward the antifungal activity of amphotericin B; as well as invite researchers to study in greater detail the biological effects of its storage and pharmaceutical administration in the absence or presence of light.

\section{Acknowledgements}

We gratefully acknowledge the thematic network of collaboration between academic groups UVCA-354 and PRODEP 2015.

\section{References}

1. M. E. Medina, A. Galano and J. R. Alvarez-Idaboy, Phys. Chem. Chem. Phys., 2015, $17,4970$.

2. M. E. Medina, A. Galano and A. Trigos, J. Phys. Chem. B , 2018,122 , 7514.

3. A. Galano and J. R. Alvarez-Idaboy, Phys. Chem. Chem. Phys., 2012, 14, 12476.

4. J. Wang, J. Z. Li, A. X. Lu, K. F. Zhang and B. J. Li, Oncol. Lett., 2014, 7, 1159.

5. F. Torres, A. González-Candia, C. Montt, G. Ebensperger, M. Chubretovic, M. Serón- Ferre, R. V. Reyes, A. J. Llanos and E. A. Herrera, J. Phys. Chem. B , 2018, 122, 6198.

6. M. Matsuda and I. Shimomura, Rev. Endocr. Metab. Disord., 2014,15 , 1.

7. A. Rahal, A. Kumar, V. Singh, B. Yadav, R. Tiwari, S. Chakraborty and K. Dhama, BioMed Res. Int., 2014, 761264.

8. C. Schweitzer, R. Schmidt, Chem. Rev., 2003, 103, 1685.

9. C. Espinoza, A. Trigos, M. E. Medina, J. Phys. Chem. A, 2016,120 , 6103.

10. A. N. Tevyashova, E. N. Olsufyeva, S. E. Solovieva, S. S. Printsevskaya, M. I. Reznikova, A. S. Trenin, O. A. Galatenko, I. D. Treshalin, E. R. Pereverzeva, E. P. Mirchink, E. B. Isakova, S. B. Zotchev and M. N. Preobrazhenskayaa, Antimicrob. Agents Chemother., 2013, 57 (8), 3815.

11. E. Grela, M. Piet, R. Luchowski, W. Grudzinski, R. Paduch and W. I. Gruszecki, Sci. Rep., 2018, 8 , 14067.

12. E. Grela, M. Wieczor, R. Luchowski, J. Zielinska, A. Barzycka, W. Grudzinski, K. Nowak, P. Tarkowski, J. Czub and W. I. Gruszecki,Mol. Pharmaceutics , 2018, 15, 4202.

13. M. Baginskia, H. Resatb and E. Borowski, Biochim. Biophys. Acta, 2002, 1567, 63.

14. M. Baran, E. Borowski and J. Mazerski, Biophys. Chem., 2009,141 (2-3), 162.

15. P. Kovacic and A. Cooksy, Med. Chem. Commun., 2012, 3, 274.

16. G. Blum, S. Perkhofer, H. Haas, M. Schrettl, R. Wurzner, M. P. Dierich and C. Lass-Florl, Antimicrob. Agents Chemother., 2008,52 (4), 1553.

17. R. Pandey, S. Mehrotra, R. S. Ray, P. C. Joshi and R. K. Hans,Drug Chem. Toxicol. , 2002, 25 (2), 215.

18. A. C. Mesa-Arango, N. Trevijano-Contador, E. Román, R. Sánchez-Fresneda, C. Casas, E. Herrero, J. C. Argüelles, J. Pla, M. Cuenca-Estrella and O. Zaragoza. Antimicrob. Agents Chemother., 2014, 58 (11), 6627 .

19. A. Trigos and A. Ortega-Regules, Mycologia, 2002,94 (4), 563.

20. M. E. Medina, A. Galano, A. Trigos, J. Phys. Org. Chem., 2015,28, 504.

21. M. E. Medina, C. Iuga, A. Trigos, J. Phys. Org. Chem. , 2016,29 , 196.

22. M. J. Frisch, G. W. Trucks, H. B. Schlegel, G. E. Scuseria, M. A. Robb, J. R. Cheeseman, G. Scalmani, V. Barone, G. A. Petersson, H. Nakatsuji, X. Li, M. Caricato, A. V. Marenich, J. Bloino, B. G. Janesko, R. Gomperts, B. Mennucci, H. P. Hratchian, J. V. Ortiz, A. F. Izmaylov, J. L. Sonnenberg, D. WilliamsYoung, F. Ding, F. Lipparini, F. Egidi, J. Goings, B. Peng, A. Petrone, T. Henderson, D. Ranasinghe, V. G. Zakrzewski, J. Gao, N. Rega, G. Zheng, W. Liang, M. Hada, M. Ehara, K. Toyota, R. Fukuda, J. Hasegawa, M. Ishida, T. Nakajima, Y. Honda, O. Kitao, H. Nakai, T. Vreven, K. Throssell, J. A. Montgomery, Jr., J. E. Peralta, F. Ogliaro, M. J. Bearpark, J. J. Heyd, E. N. Brothers, K. N. Kudin, V. N. Staroverov, T. A. Keith, R. Kobayashi, J. Normand, K. Raghavachari, A. P. Rendell, J. C. Burant, 
S. S. Iyengar, J. Tomasi, M. Cossi, J. M. Millam, M. Klene, C. Adamo, R. Cammi, J. W. Ochterski, R. L. Martin, K. Morokuma, O. Farkas, J. B. Foresman, and D. J. Fox. Gaussian 16 , Revision A.03, Gaussian, Inc., Wallingford CT, 2016.

23. Y. Zhao, D. G. Truhlar, Theor. Chem. Acc., 2008, $120,215$.

24. A. V. Marenich, C. J. Cramer, D. G. Truhlar, J. Phys. Chem. B , 2009, 113 , 6378.

25. Y. Zhao, D. G. Truhlar, J. Phys. Chem. A, 2008, $112,1095$.

26. A. Galano, J. R. Alvarez-Idaboy, J. Comput. Chem. , 2014,35 , 2019.

27. H. Eyring, J. Chem. Phys. , 1935, 3, 107.

28. D. G. Truhlar, B. C. Garrett, S. J. Klippenstein, J. Phys. Chem. , 1983, 87, 2664.

29. R. A. Marcus, Annu. Rev. Phys. Chem., 1964, 15, 155.

30. R. A. Marcus, Rev. Mod. Phys., 1993, 65, 599.

31. R. A. Marcus, Pure Appl. Chem., 1997, 69, 13.

32. S. F. Nelsen, M. N. Weaver, Y. Luo, J. R. Pladziewicz, L. K. Ausman, T. L. Jentzsch, J. J. OKonek, J. Phys. Chem. A, 2006,110, 11665 .

33. F. C. Collins, G. E. Kimball, J. Colloid Sci. , 1949,4 , 425.

34. M. Z. Smoluchowski, Phys. Chem. , 1917, 92, 129.

35. D. G. Truhlar, J. Chem. Educ., 1985, $62,104$.

36. A. Einstein, Ann. Phys. (Berlin, Ger.), 1905, $\mathbf{3 2 2}, 549$.

37. G. G. Stokes, 1903 Mathematical and Physical Papers ; Cambridge University Press: Cam- bridge, U.K., 1903, Vol. 3 (esp. section IV).

38. A. Galano, J. R. Alvarez-Idaboy, J. Comput. Chem., 2013,34, 2430.

Y. Chang, Y.-H. Wang, C.-Q. Hu, J. Antibiot. , 2011, $64,735$. 